Trauma Berufskrankh 2003 - 5 [Suppl 2]: $\$ 296-\$ 304$ DOI 10.1007/s10039-002-0660-5
Septische Chirurgie

Thomas Mückley · Martin Kirschner · Christian Hierholzer · Gunther 0. Hofmann

BG-Unfallklinik Murnau

\section{Spondylitis - Spondylodiszitis}

Neue Therapiekonzepte

\section{Zusammenfassung}

Die Inzidenz der Spondylitis/Spondylodiszitis nimmt aufgrund multifaktorieller Ursachen, jedoch wesentlich aufgrund der AidsEpidemie, der großen Anzahl der i. v. Drogenabhängigen, dem Wiederauftreten der Tuberkulose in den Industriestaaten und der zunehmende Anzahl an immunsupprimierten Patienten zu. Frühstadien ohne Lähmungssymptomatik und ohne wesentliche Instabilität können konservativ mit Bettruhe und testgerechter Antibiotikatherapie erfolgreich behandelt werden. Neu aufgetretene neurologische Ausfälle und septische Verläufe stellen chirurgische Notfälle dar. Bei ausgedehnten knöchernen Destruktionen ist die konservative Ausheilungschance gering. Sie bedeutet eine langzeitige Immobilisierung mit ungewissem Behandlungserfolg. Des Weiteren stellen Abszessbildungen, Instabilitäten, Fehlstellungen der Wirbelsäule und eine drohende Querschnittsymptomatik Grenzen der konservativen Therapie dar.Ziele der operativen Therapie sind die Resektion des Infektherds und die Stabilisierung der Wirbelsäule mit Korrektur bestehender Fehlstellungen. Bei der Rekonstruktion werden winkelstabile Implantate und Titankörbe als Wirbelkörperersatz eingesetzt. Minimalinvasive Operationstechniken gewinnen zunehmend an Verbreitung. Um fortgeschrittene Stadien der Spondylitis/Spondylodiszitis mit entsprechenden Komplikationen zu vermeiden sind eine rechtzeitige Diagnosestellung und Therapieeinleitung unabdingbar.

\section{Schlüsselwörter}

Spondylodiszitis - Therapiekonzept · Wirbelkörperosteomyelitis - Endoskopische Wirbelsäulenchirurgie

as Krankheitsbild der bakteriellen Entzündung der Wirbelsäule wurde erstmals 1879 durch Lannelongue [11] und 1936 ausführlich durch Kulowski [10] beschrieben. Als Ursache wurde bereits damals die hämatogene Streuung von Bakterien vermutet [10]. Begrenzt sich die Entzündung auf den Wirbelkörper, wird von einer Spondylitis bzw. Wirbelkörperosteomyelitis gesprochen. Greift die entzündliche Erkrankung auf die benachbarte Bandscheibe über, wird sie als Spondylodiszitis bezeichnet. Die alleinige Entzündungen der Bandscheibe (Diszitis) ist beim Erwachsenen selten und tritt dann v. a. sekundär nach operativen Eingriffen im Bandscheibenfach auf.

\section{Inzidenz und Ätiologie}

Die Inzidenz der Spondylitis/Spondylodiszitis ist gering und wurde auf 1:250.000 Einwohner pro Jahr eingeschätzt [4]. Ihr Anteil an allen infektiösen Knochenerkrankungen beträgt lediglich 2-4\% [24]. Während die Mortalitätsrate in der ersten Hälfte des 20. Jahrhunderts noch zwischen 40 und 90\% lag, konnte sie im Ausgang des letzten
Jahrhunderts auf unter $5 \%$ gesenkt werden [5]. Trotzdem bedarf die Spondylitis/Spondylodiszitis großer Beachtung, da sie zu erheblichen Destruktionen und Profilstörungen der Wirbelsäule mit persistierenden Schmerzzuständen und neurologischen Ausfällen führen kann. Des Weiteren stellt sie eine schwere, konsumierende Erkrankung dar, die bei entsprechend reduziertem Allgemeinzustand vital bedrohlich ist.

Die Ätiologie hat sich im Verlauf der Jahrzehnte deutlich gewandelt. Das frühere Überwiegen spezifischer Entzündungen hat sich in den letzten Jahrzehnten zugunsten unspezifischer bakterieller Infektionen des Wirbelkörpers und seiner angrenzenden Strukturen verschoben [2]. Bei den pyogenen Entzündungen werden am häufigsten Staphylokokken nachgewiesen. In den letzten Jahren wird über eine erneute Veränderung des Erregerspektrums durch das Wiederauftreten der Tuberkulose in den Industriestaaten und durch gehäufte gramnegative Infektionen bei i.-v.-Drogenabhängigen berichtet.

\footnotetext{
(c) Springer-Verlag 2003

Dr.Thomas Mückley BG-Unfallklinik Murnau, Prof.-Küntscher-Straße 8, 82418 Murnau,

E-Mail:T.Mueckley@gmx.de, Phone: 08841-482572, Fax: 08841-482203
} 
T. Mückley $\cdot$ M. Kirschner $\cdot$ C. Hierholzer . G. O. Hofmann

\section{Spondylitis - Spondylodiscitis. New concepts in surgical treatment}

\begin{abstract}
The incidence of spondylitis/spondylodiscitis is increasing and has been for some decades. Some of the multiple causative factors in this increase are the HIV epidemic, the large number of intravenous drug abusers, and the recurrence of tuberculosis in the industrialized countries. Early stages of spondylitis/spondylodiscitis without neurologic deficit or spinal instability can be successfully treated by conservative means with immobilization and appropriate antibiotics. Neurologic deficit and sepsis are surgical emergencies and require immediate operative treatment. When there is extensive bony destruction, the prognosis with conservative treatment is poor, including that of longterm immobilization with which success is uncertain. Abscess formation, spinal instability and spinal deformity also require operative treatment. Surgical treatment includes resection of the infectious focus and restoration of spinal stability with correction of deformity. Implants for spinal reconstruction consist of angle-stable plates and titanium baskets for vertebral replacement. Currently, minimally invasive operative techniques are emerging. Essential factors in successful treatment of spondylitis/spondylodiscits are early diagnosis and onset of treatment to avoid progressive stages with corresponding complications.
\end{abstract}

\section{Keywords}

Spondylodiscitis - Therapeutic algorithm . Vertebral osteomyelitis - Endoscopic spine surgery

\section{Diagnostik und Therapie}

Die klinischen Symptome der Spondylitis/Spondylodiszitis sind meist uncharakteristisch, was häufig zu einer Verzögerung der Diagnosestellung führt. Bei 2/3 der Patienten bestehen die Symptome zum Zeitpunkt der Diagnosestellung bereits länger als 3 Monate [7]. Diese Verzögerung der Diagnostik und Therapie muss als das zentrale Problem in der Behandlung der Spondylodiszitis angesehen werden, da sie das Fortschreiten der Wirbelkörperdestruktion und das Auftreten von neurologischen Komplikationen, segmentalen Instabilitäten und späteren Fehlstellungen begünstigt.

Ein wesentlicher Baustein der Therapie ist die erregerspezifische Antibiotikatherapie. Das weitere Vorgehen wird kontrovers diskutiert. Basierend auf guten Ergebnissen bevorzugen viele Autoren die konservative Therapie. Diese besteht neben der Antibiotikatherapie aus einer konsequenten Ruhigstellung der Wirbelsäule mit Bettruhe über mehrere Wochen und nachfolgender Mobilisation mit Stützkorsett oder Gipsmieder. Komplikationen der konservativen Therapie sind die Pseudarthrosenbildung, die Instabilität der betroffenen Segmente, die zunehmende kyphotische Deformität, chronische Schmerzzustände, neurologische Ausfälle und Abszessformationen [15]. Treten die beschriebenen Komplikationen im Rahmen der konservativen Therapie auf, wird ein chirurgisches Vorgehen empfohlen. Weitere Operationsindikationen sind septische Verläufe, fortschreitende Wirbelkörperdestruktionen, persistierende Beschwerden nach konservativer Therapie und Vorerkrankungen des Patienten, die keine längere Immobilisation zulassen. Die operative Behandlung erfolgt mit dem Ziel der Sanierung des Entzündungsherds, der Stabilisierung der destruierten Wirbelsäulenabschnitte und der Dekompression des Myelons bzw. der Kauda.

Im Folgenden wird anhand von Fallbeispielen über neue Therapiekonzepte in der Behandlung der Spondylitis/Spondylodiszitis berichtet.

\section{Kasuistik}

\section{Fall 1}

Anamnese

Der 55-jährige Mann litt nach einem Bagatelltrauma an zunehmenden Rückenschmerzen im LWS-Bereich. Der Hausarzt führte eine Schmerztherapie mit NSAID und lokalen Infiltrationen durch. Als Vorerkrankungen bzw. Risikofaktoren bestanden eine Psoriasis vulgaris, ein chronischer Nikotin- und Alkoholabusus sowie eine in der Jugend durchlittene Lungentuberkulose. Von den ersten Symptomen bis zur stationären Einweisung vergingen 6 Monate.

\section{Befund}

Bei der Untersuchung zeigte sich der Patient im deutlich reduzierten Allgemeinzustand mit Ruhe- und Klopfschmerzen im Brust-Lendenwirbelsäulen-Übergang ohne neurologische Ausfälle. Es bestanden subfebrile Temperaturen.

Die Laborchemie ergab: Leukozyten 11,3/nl, CRP 6,7 mg/dl, BKS 72/>100 mm. Die konventionelle Röntgen- und CT-Diagnostik der LWS zeigte eine Spondylodiszitis BWK12/LWK1 mit ausgeprägter Wirbelkörperdestruktion und linksseitigem Psoasabszess (Abb. 1a,b).

\section{Verlauf}

Am Aufnahmetag wurde eine linksseitige Minilumbotomie mit Entlastung des Psoasabszesses, Débridement, Wundabstrich und Dränage durchgeführt. Bei klinischem Verdacht auf einen tuberkulösen Prozess wurde initial mit einer tuberkulostatischen 3 fach-Therapie (Rifampicin, Isoniazid, Ciprofloxacin) begonnen. Nach 4 Wochen konnte die extrapulmonale Tuberkulose anhand einer Mykobakterienkultur bestätigt werden. Wegen der fortgeschrittenen Wirbelkörperdestruktion mit kyphotischer Fehlstellung wurden eine dorsale Stabilisierung mit Fixateur interne, die Teilkorporektomie der betroffenen Wirbelkörper, der Wirbelkörperersatz mit Titankorb und eine ventrale winkelstabile Stabilisierung mit Stabsystem durchgeführt (Abb. 1c). Der weitere Verlauf gestaltete sich unkompliziert, und der Patient wurde ohne Korsett mobilisiert. Die stationäre Behandlung erstreckte 


\section{Septische Chirurgie}
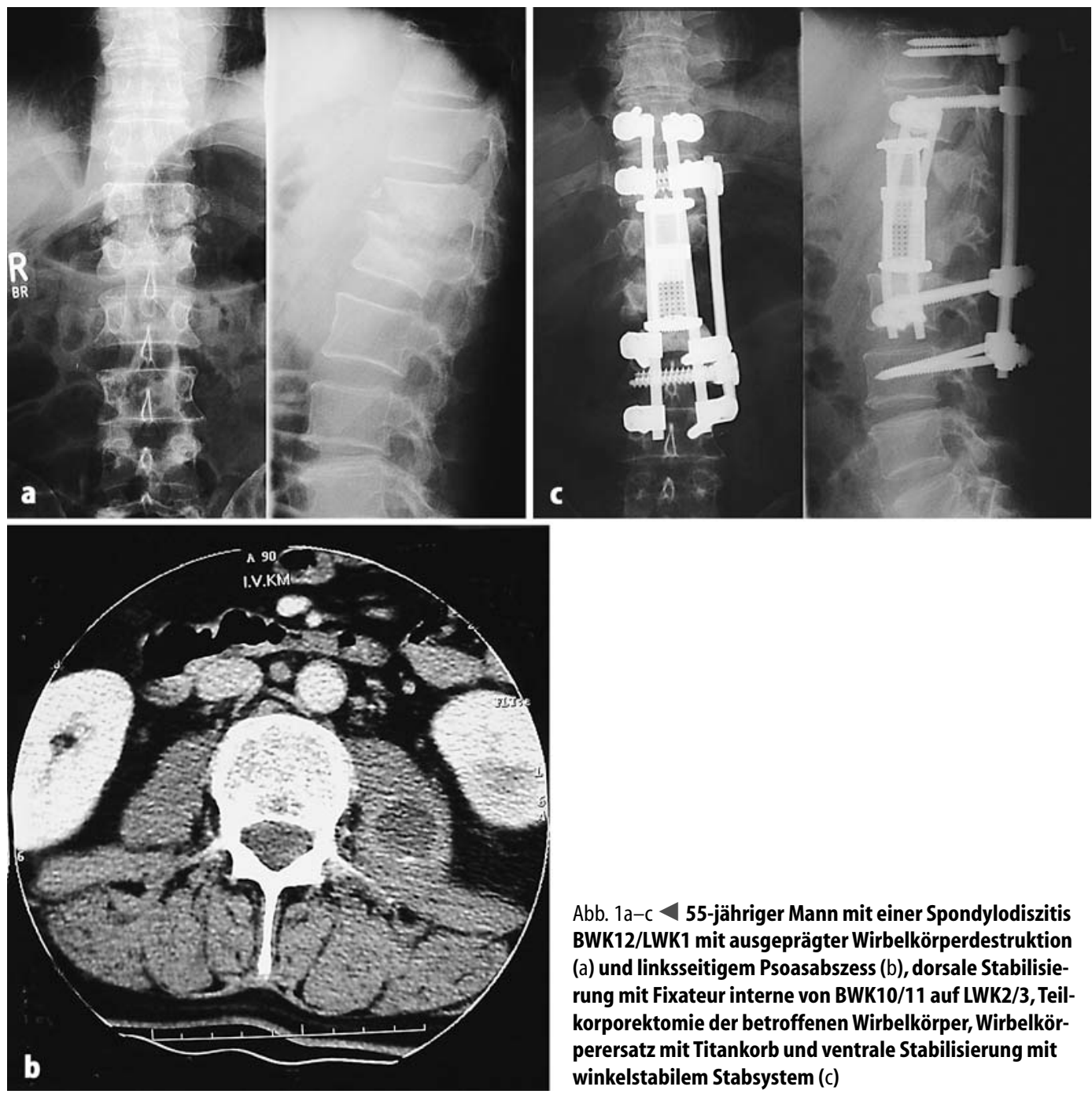

Abb. 1a-c $<$ 55-jähriger Mann mit einer Spondylodiszitis BWK12/LWK1 mit ausgeprägter Wirbelkörperdestruktion (a) und linksseitigem Psoasabszess (b), dorsale Stabilisierung mit Fixateur interne von BWK10/11 auf LWK2/3, Teilkorporektomie der betroffenen Wirbelkörper, Wirbelkörperersatz mit Titankorb und ventrale Stabilisierung mit winkelstabilem Stabsystem (c)

sich über 3 Monate. Die tuberkulostatische Therapie wird nach der Entlassung fortgeführt.

\section{Fall 2}

\section{Anamnese}

Bei dem 72-jährigen Mann bestanden seit 5 Monaten Schmerzen im Bereich der LWS, weswegen lokale Infiltrationen durchgeführt wurden. Im Verlauf kam es $\mathrm{zu}$ einem plötzlichen Harnverhalt mit sofortiger Einweisung in eine urologische Fachabteilung. Nach der Aufnahme wurde der Patient zunehmend somnolent und musste intubiert werden. Die durchgeführte CT-Diagnostik ergab eine Spondylodiszitis LWK4/5 mit epiduraler Abszedierung und linksseitigem Psoasabszess (Abb. 2). Der Patient wurde nachfolgend in unsere Klinik verlegt.

\section{Befund}

In der Notaufnahme fand sich ein analgosedierter, intubierter, beatmeter und katecholaminpflichtiger Patient. Die Kör- pertemperatur betrug $39^{\circ} \mathrm{C}$. Die Labordiagnostik ergab: Leukozyten $9,4 / \mathrm{nl}$, CRP 20,9 mg/dl, Thrombozyten 77.000/ $\mu$ l, Quick 54\%, PTT 67 s.

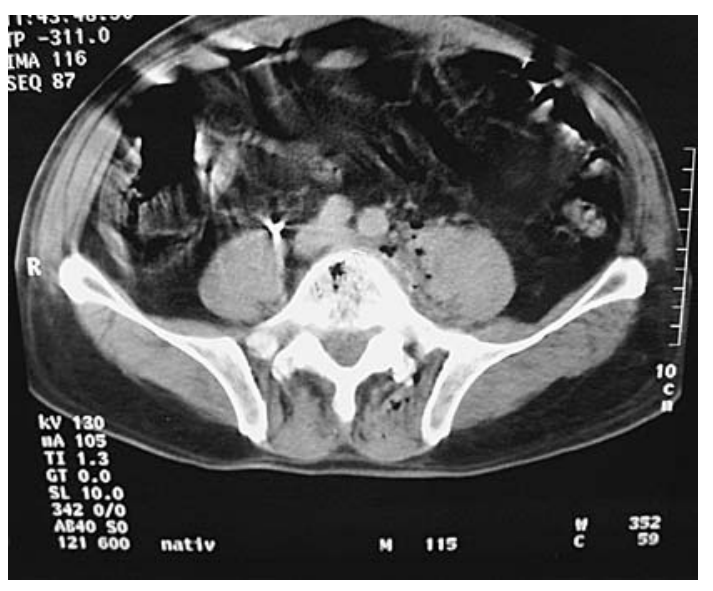

Abb. $2<72$-jähriger Mann mit einer Spondylodiszitis LWK4/5 mit epiduraler Abszedierung, linksseitigem Psoasabszess mit Gasbläschen und Mitbeteiligung der dorsalen Rückenmuskulatur 

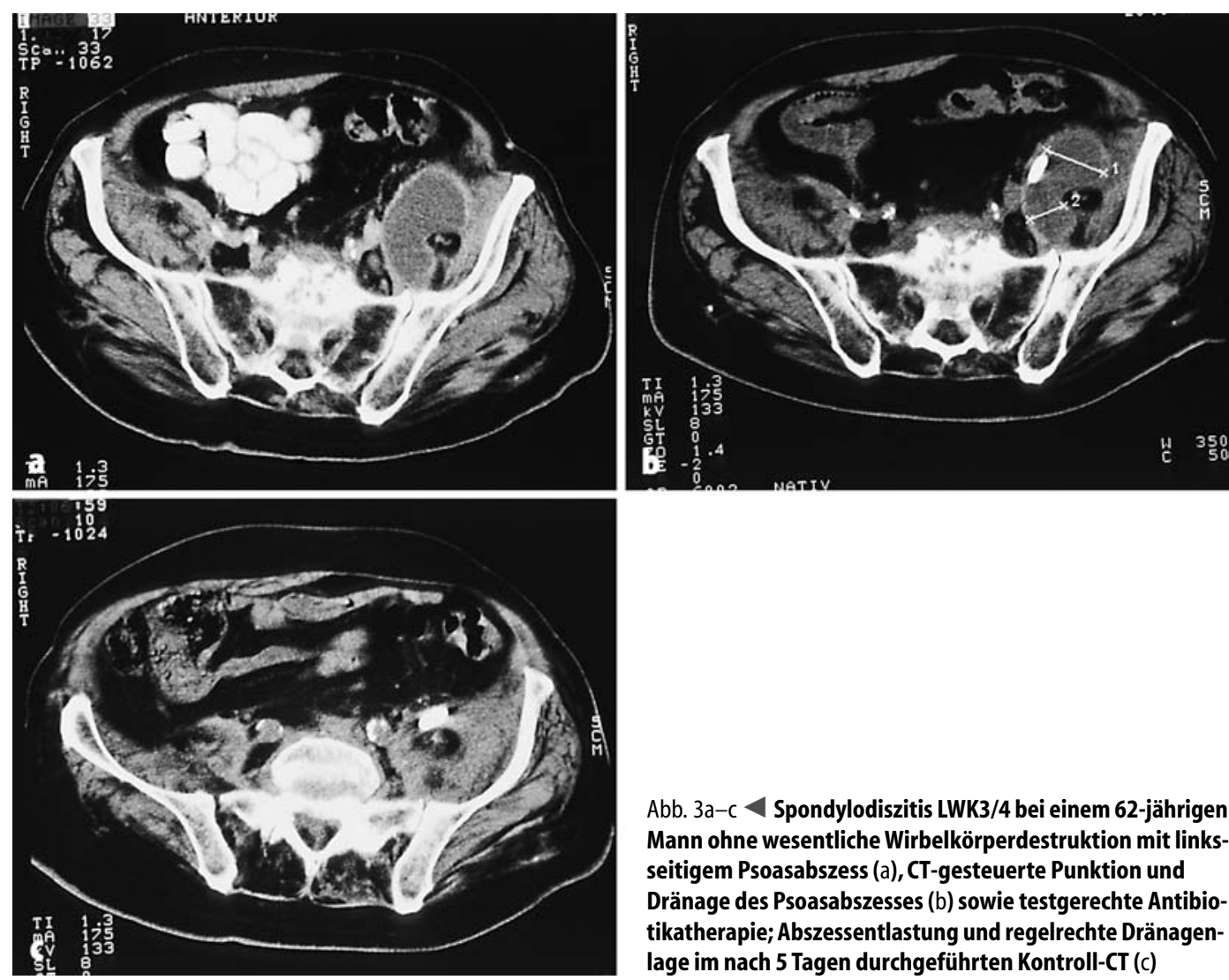

Abb. 3a-c 4 Spondylodiszitis LWK3/4 bei einem 62-jährigen Mann ohne wesentliche Wirbelkörperdestruktion mit linksseitigem Psoasabszess (a), CT-gesteuerte Punktion und Dränage des Psoasabszesses (b) sowie testgerechte Antibiotikatherapie; Abszessentlastung und regelrechte Dränagenlage im nach 5 Tagen durchgeführten Kontroll-CT (c)

Verlauf

Nach Stabilisierung der Gerinnung erfolgte eine linksseitige Lumbotomie mit Débridement, Spülung und Dränage. Intraoperativ zeigten sich massive Nekrose- und Abszessareale des linksseitigen M. psoas. In gleicher Sitzung wurden nach Umlagerung in Bauchlage über einen dorsalen Zugang das Débridement der betroffenen Rückenmuskulatur und eine Entlastung des epiduralen Abszesses mit Teillaminektomie vorgenommen. Auf der Intensivstation wurde eine Liquorpunktion in Höhe LWK2 durchgeführt. Die mikrobiologische Untersuchung ergab Escherichia coli. Eine Kombinationstherapie aus Metronidazol, Cefepim, Netilmicin und Chloramphenicol wurde noch am Aufnahmetag angesetzt. 3 weitere geplante operative Revisionen mit Débridements, Spülung und Vakuumversiegelung folgten. Begleitend wurde die hyperbare Sauerstofftherapie (HBO) nach dem Problemwundenschema $(3 \times 30 \mathrm{~min}$, Sauerstoffatmung unter $1,4 \times 10^{5} \mathrm{~Pa}$ Überdruck mit $2 \times 10 \mathrm{~min}$ Sauerstoffpause) eingesetzt. Trotz Maximaltherapie verstarb der Patient nach $23 \mathrm{Ta}$ gen im septischen Multiorganversagen.
Fall 3

\section{Anamnese}

Der 62-jährige Mann litt seit 3 Monaten an Schmerzen im LWS-Bereich. Anamnestisch waren seit Jahren ein insulinpflichtiger Diabetes mellitus, eine Herzinsuffizienz, eine chronisch-obstruktive Lungenerkrankung sowie ein Alkoholund Nikotinabusus zu erheben. Vorausgegangen waren mehrfache lokale Infiltrationen wegen der Rückenschmerzen. Eine linksseitige Beinschwellung mit massiven Schmerzen beim Gehen führte $\mathrm{zu}$ einer auswärtigen stationären internistischen Behandlung, bei der eine Thrombose ausgeschlossen wurde. Nach kurzem Aufenthalt zu Hause kam es zu einer deutlichen Beschwerdezunahme und zur Einweisung in unsere Klinik.

\section{Befund}

Es zeigte sich ein stark übergewichtiger Patient in reduziertem Allgemeinzustand. Es bestand ein Ruheschmerz im Bereich der LWS mit Ausstrahlung in das linke Bein. Die Labordiagnostik ergab: Leukozyten 13,7/nl, CRP 14,9 mg/dl. Die kon- ventionelle Röntgen- und CT-Diagnostik zeigte eine Spondylodiszitis LWK 3/4 ohne wesentliche Wirbelkörperdestruktion mit linksseitigem Psoasabszess (Abb. 3a).

\section{Verlauf}

Nach entsprechender Vorbereitung erfolgten die CT-gesteuerte Punktion und Dränage des Psoasabszesses (Abb. 3b). Die mikrobiologische Untersuchung des Punktats ergab Staphylococcus aureus. Aufgrund der erheblichen Vorerkrankungen wurde auf operative Maßnahmen verzichtet. Eine testgerechte Antibiotikatherapie mit Clindamycin wurde verordnet. Eine nach 5 Tagen durchgeführte Kontrollcomputertomographie zeigte die Abszessentlastung und die regelrechte Dränagenlage (Abb. 3c). Nach Rückgang der Beschwerdesymptomatik und Normalisierung der Entzündungsparameter wurden die Dränage entfernt und der Patient mit einem Stützkorsett versorgt mobilisiert. Der stationäre Aufenthalt dauerte 14 Tage. Die Antibiose wurde für 6 Wochen, die Mobilisierung mit Stützkorsett für 3 Monate fortgeführt. 


\section{Septische Chirurgie}
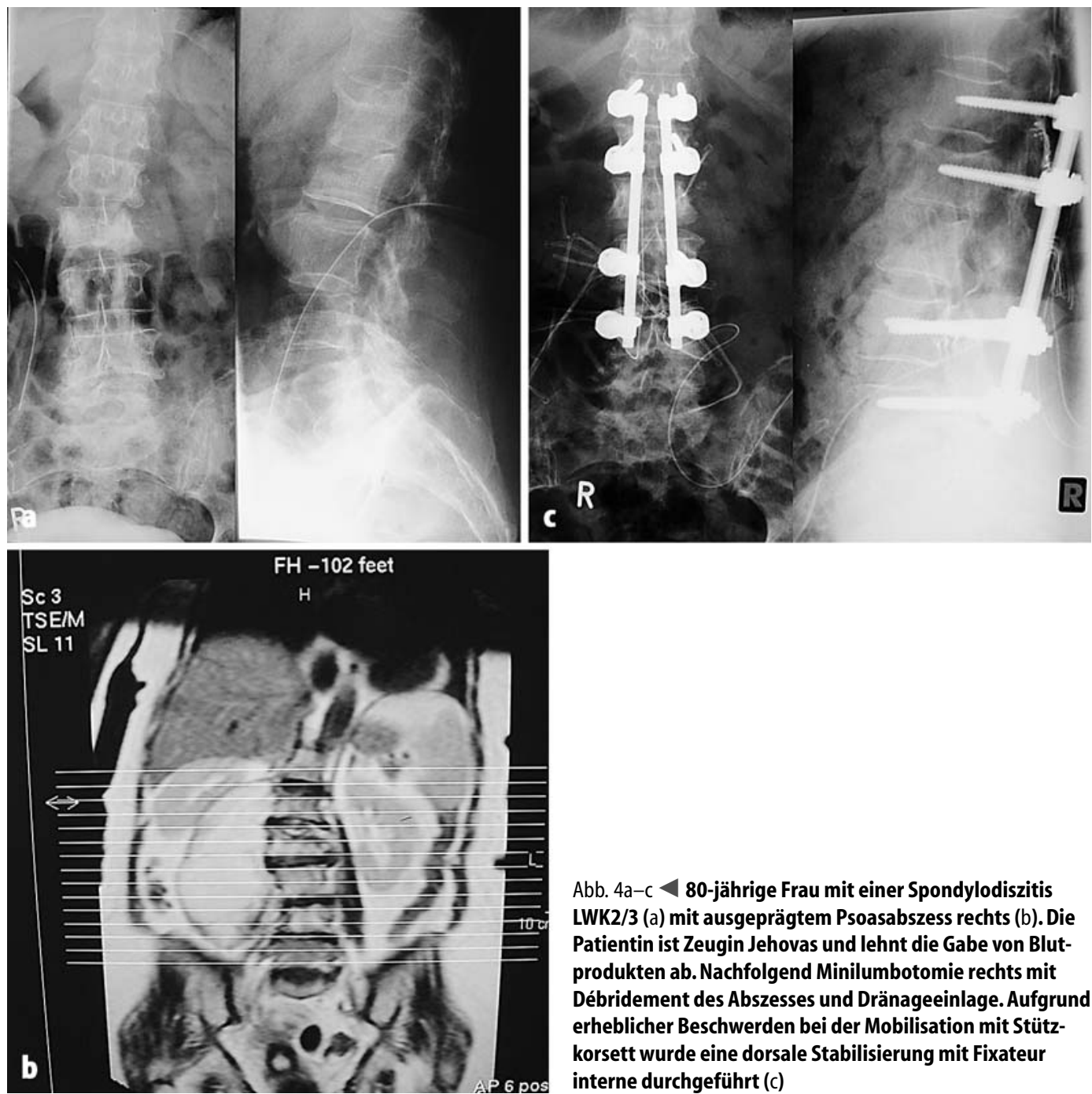

Abb. 4a-c $<$ 80-jährige Frau mit einer Spondylodiszitis LWK2/3 (a) mit ausgeprägtem Psoasabszess rechts (b). Die Patientin ist Zeugin Jehovas und lehnt die Gabe von Blutprodukten ab. Nachfolgend Minilumbotomie rechts mit Débridement des Abszesses und Dränageeinlage. Aufgrund erheblicher Beschwerden bei der Mobilisation mit Stützkorsett wurde eine dorsale Stabilisierung mit Fixateur interne durchgeführt (c)

\section{Fall 4}

\section{Anamnese}

Bei der 80-jährigen Frau mit rezidivierenden Lumboischialgien in der Vorgeschichte bestanden seit 2 Wochen therapieresistente Rückenschmerzen im Bereich der Lendenwirbelsäule, die zur stationären Einweisung führten. Am 5. Tag der stationären Behandlung wurden eine konventionelle Röntgenaufnahme und eine Kernspintomographie durchgeführt, welche eine Spondylodiszitis LWK2/3 mit ausgeprägtem Psoasabszess rechts darstellten (Abb. 4a,b). In der Anamnese waren eine KHK und eine kompensierte Niereninsuffizienz bekannt. Die Patientin ist Zeugin Jehovas und lehnt die Gabe von Blutprodukten ab. Sie wurde uns zur weiteren Therapie zuverlegt.

\section{Befund}

Es zeigte sich eine Patientin in deutlich reduziertem Allgemeinzustand mit Ruhe- und Belastungsschmerzen im LWSBereich, welche in das rechte Bein ausstrahlten. Neurologische Ausfälle bestanden nicht. Die Labordiagnostik ergab: Leukozyten 13,o/nl, CRP 6,3 mg/dl.

\section{Verlauf}

Nach entsprechender Vorbereitung erfolgte die Minilumbotomie rechts mit Débridement des Abszesses und Dränageeinlage. In der mikrobiologischen Analyse konnten Mykobakterien nachgewiesen werden. Aufgrund des Lebensalters und der bestehenden Vorerkrankungen wurde eine frühe Mobilisation der Patientin mit Stützkorsett versucht, die wegen erheblicher Rückenschmerzen ab- gebrochen werden musste. Mit der Patientin wurde eine dorsale Stabilisierung mit Fixateur interne zur besseren Mobilisierung besprochen und nachfolgend durchgeführt (Abb. 4c). Im weiteren Verlauf konnte die Patientin im Gehwagen deutlich beschwerdegebessert mobilisiert werden. Von einer Infektsanierung des betroffenen Wirbelsäulenabschnitts wurde wegen der Ablehnung einer Fremdblutgabe abgesehen. Die medikamentöse Therapie der Organtuberkulose erfolgte mit einer 4 fach-Kombination (Rifampicin, Myambutol, Isoniazid, Ciprofloxacin). Nach 7 Wochen stationärer Behandlung wurde die Patientin zur Fortführung der tuberkulostatischen Therapie in eine entsprechende Fachklinik verlegt. 

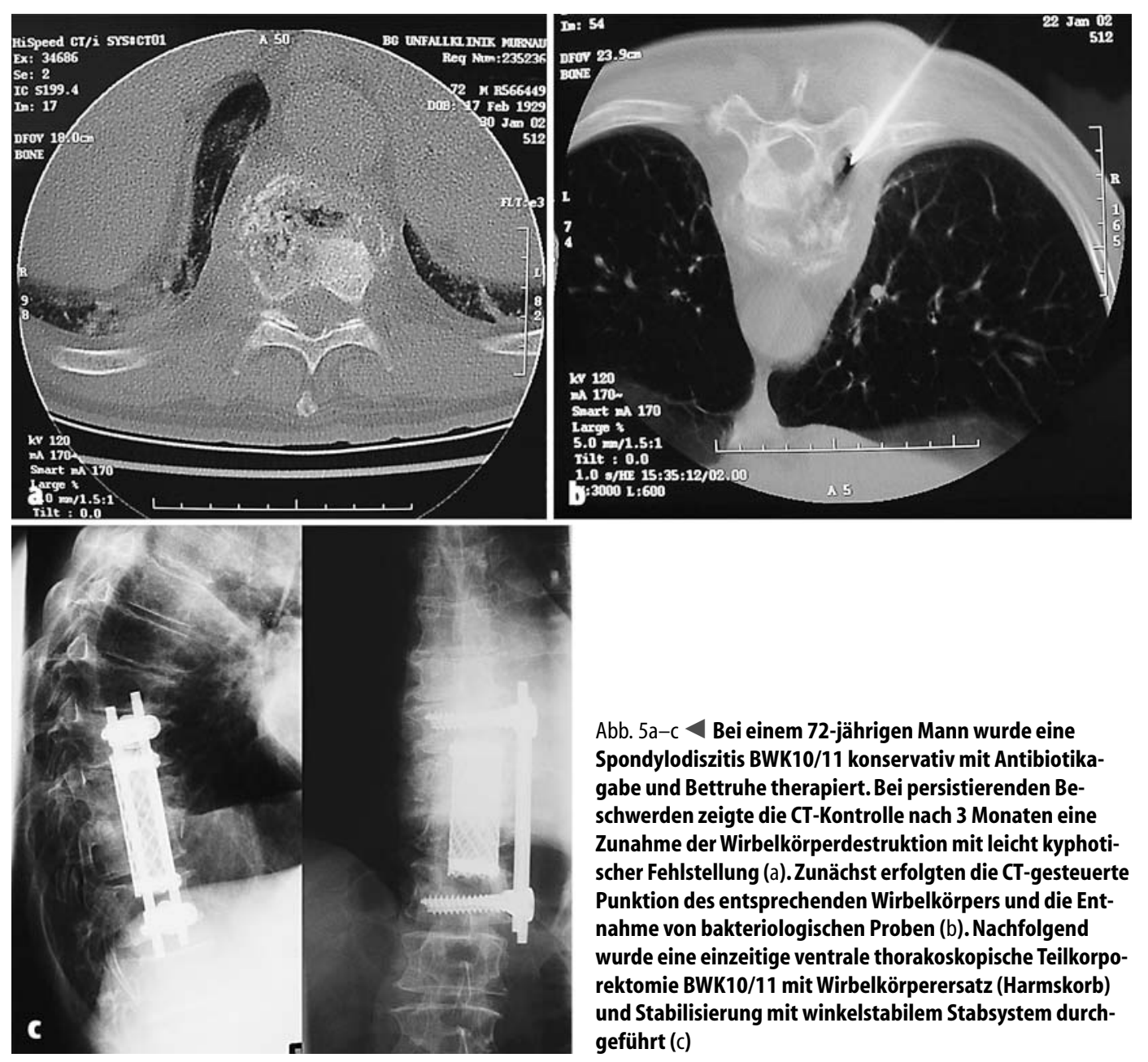

\begin{abstract}
Abb. 5a-c 4 Bei einem 72-jährigen Mann wurde eine Spondylodiszitis BWK10/11 konservativ mit Antibiotikagabe und Bettruhe therapiert. Bei persistierenden Beschwerden zeigte die CT-Kontrolle nach 3 Monaten eine Zunahme der Wirbelkörperdestruktion mit leicht kyphotischer Fehlstellung (a).Zunächst erfolgten die CT-gesteuerte Punktion des entsprechenden Wirbelkörpers und die Entnahme von bakteriologischen Proben (b). Nachfolgend wurde eine einzeitige ventrale thorakoskopische Teilkorporektomie BWK10/11 mit Wirbelkörperersatz (Harmskorb) und Stabilisierung mit winkelstabilem Stabsystem durchgeführt (c)
\end{abstract}

Fall 5

\section{Anamnese}

Bei dem 72-jährigen Mann bestanden seit mehreren Monaten Rückenschmerzen. Es wurden eine Spondylodiszitis BWK10/11 diagnostiziert und eine konservative Therapie mit Antibiotikagabe und Bettruhe eingeleitet. Mit dieser Therapie kam es über 3 Monate zu keiner Besserung der Beschwerden, und die Immobilisation führte zur deutlichen Beeinträchtigung des Allgemeinzustands des Patienten. Zur weiteren Abklärung und Therapie wurde der Patient in unsere Klinik verlegt. An Vorerkrankungen ist eine Herzinsuffizienz aufzuführen.

\section{Befund}

Der Patient in reduziertem Allgemeinzustand gab Ruhe- und Belastungsschmerzen im Bereich der unteren BWS an. Neurologische Ausfälle lagen nicht vor. In der Labordiagnostik wurden 11,6/nl Leukozyten und 5,0 mg/dl CRP bestimmt. Die konventionelle Röntgenund CT-Diagnostik zeigte eine Spondylodiszitis BWK10/11 mit deutlicher Wirbelkörperdestruktion und leichter kyphotischer Fehlstellung (Abb. 5a).

\section{Verlauf}

Zunächst erfolgten die CT-gesteuerte Punktion des entsprechenden Wirbelkörpers und die Entnahme von bakteriologischen Proben (Abb. 5b). Die mikrobiologische Untersuchung ergab Propionibacterium spec. Eine testgerechte Antibiotikatherapie mit Linezolid wurde eingeleitet. Nach entsprechender Aufklärung des Patienten über die möglichen Therapieoptionen wurde eine einzeitige ventrale thorakoskopische Teilkorporektomie BWK10/11 mit Débridement, Wirbelkörperersatz mit Harmskorb und ventraler Stabilisierung mit winkelstabilem Stabsystem durchgeführt
(Abb. 5c). Der peri- und postoperative Verlauf waren unkompliziert. Der Patient wurde ohne Stützkorsett mobilisiert und nach 4 Wochen stationärer Behandlung deutlich beschwerdegebessert zur AHB-Maßnahme entlassen.

\section{Fall 6}

\section{Anamnese}

Bei der 57-jährigen Frau wurde in der Vorgeschichte eine Kniegelenkendoprothese rechts implantiert. In der Folge kam es zur Protheseninfektion mit mehreren operativen Revisionen, zum Prothesenausbau und zur Kniegelenkarthrodese mittels Fixateur externe. Eine Woche später traten gürtelförmige Beschwerden im unteren BWS-Bereich mit erhöhten Entzündungsparametern in der Labordiagnostik auf, und die Patientin wurde in eine urologische Fachabteilung mit dem Verdacht auf eine Pyelonephritis verlegt. Eine Antibiotikatherapie wur- 


\section{Septische Chirurgie}
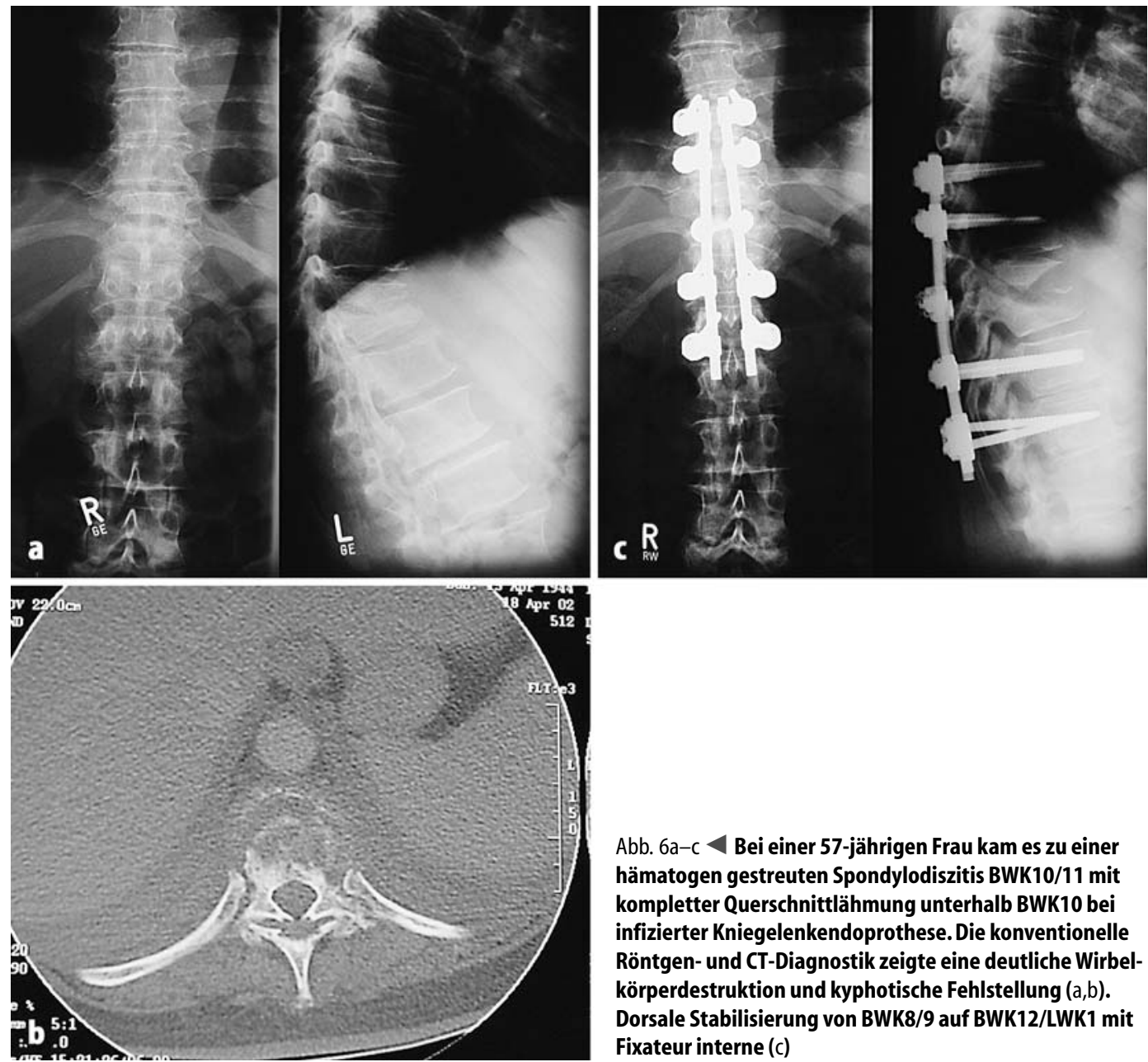

Abb. 6a-c 4 Bei einer 57-jährigen Frau kam es zu einer hämatogen gestreuten Spondylodiszitis BWK10/11 mit kompletter Querschnittlähmung unterhalb BWK10 bei infizierter Kniegelenkendoprothese. Die konventionelle Röntgen- und CT-Diagnostik zeigte eine deutliche Wirbelkörperdestruktion und kyphotische Fehlstellung $(a, b)$. Dorsale Stabilisierung von BWK8/9 auf BWK12/LWK1 mit Fixateur interne (c)

de eingeleitet. Eine urologische Erkrankung als Ursache für die bestehenden Beschwerden konnte ausgeschlossen werden. Nach 2 Wochen stationärer Behandlung wurde bei weiterhin stark erhöhten Entzündungsparametern (CRP $21 \mathrm{mg} / \mathrm{dl}$, Leukozyten $13,3 / \mathrm{nl}$ ) und $39,9^{\circ} \mathrm{C}$ Körpertemperatur eine MRT der Wirbelsäule durchgeführt. Hier zeigte sich eine Spondylodiszitis BWK10/11. Im weiteren Verlauf bildete sich eine komplette Querschnittlähmung unterhalb BWK10 aus. Auf eine operative Intervention wurde verzichtet. Bei einer CT-gesteuerten Biopsie gelang kein Erregernachweis. Die Patientin wurde uns 5 Wochen nach Ausbildung der Paraplegie zur Therapie von ausgeprägten präsakralen Ulzera und zur weiteren Rehabilitation zuverlegt.

\section{Befund}

Bei der massiv übergewichtigen, bettlägrigen Patientin bestand ein großes infiziertes präsakrales Ulkus. Ein Fixateur externe lag kniegelenküberbrückend am rechten Bein an. Die konventionelle Röntgen- und CT-Diagnostik zeigte eine Spondylodiszitis BWK1o/11 mit deutlicher Wirbelkörperdestruktion und kyphotischer Fehlstellung (Abb. 6a,b).

\section{Verlauf}

Zunächst wurde das präsakrale Ulkus mehrmals operativ débridiert und abschließend der Weichteildefekt mit einer Verschiebeschwenklappenplastik gedeckt. Nachfolgend wurde zur Stabilisierung der Wirbelsäule eine dorsale Instrumentation mit Fixateur interne durchgeführt (Abb. 6c). Eine aufgetretene respiratorische Insuffizienz komplizierte den Verlauf und erforderte eine intensivmedizinische Therapie. Die Antibiotikatherapie erfolgte mit Linezolid ohne bisherigen Keimnachweis. Nach 2-wöchiger Intensivtherapie konnte die Patientin zur weiteren Rehabilitation in eine spezialisierte Abteilung in unserer
Klinik verlegt werden. Die Therapie dauert derzeit an.

\section{Diskussion}

\section{Diagnose}

Die unspezifische Beschwerdesymptomatik der Spondylitis/Spondylodiszitis führt zu einer langen Latenz zwischen den ersten Symptomen und der letztendlichen Diagnosestellung. Diese Zeitspanne beträgt in der Regel 4-6 Monate und konnte auch durch die Einführung der Schnittbildverfahren nur unwesentlich verkürzt werden [6]. Mögliche Komplikationen einer späten Diagnose umfassen

- die zunehmende Kyphose,

- die Instabilität der Wirbelsäule,

- chronische Rückenschmerzen,

- neurologische Ausfälle,

- Abszessformationen und

- septische Verläufe [15]. 
Auch laborchemisch wird die Diagnosestellung nicht wesentlich erleichtert. Die Leukozytenzahlen sind meist unregelmäßig erhöht, wohingegen das CRP und die BSG den Verlauf der entzündlichen Reaktion in der Regel adäquat widerspiegeln $[5,22]$.

Die Infektion des Wirbelkörpers beginnt mit einer Aussaat von Mikroorganismen im endplattennahen, gut durchbluteten Knochen über die zuführenden Arterien oder den Batson-Venenplexus [16]. Die frühe Entzündungsreaktion auf diese Ansiedelung von Bakterien im Wirbelkörper umfasst eine exsudative, ödematöse und ischämische Phase im Knochenmark, ohne wesentliche Veränderungen der Knochenstruktur zu erzeugen. Diese Veränderungen der Durchblutungssituation und des Flüssigkeitshaushalts können mit der Kernspintomographie vor Eintreten einer knöchernen Destruktion festgestellt werden. Veränderungen der Wirbelkörperendplatten sowie Verschmälerungen der Bandscheibenfächer sind frühestens 4-12 Wochen nach Beginn der Erkrankung auf den konventionellen Röntgenaufnahmen sowie in der Computertomographie erkennbar [20]. Die Sensitivität der Kernspintomographie beträgt statistisch $96 \%$ und die Spezifität 92\% gegenüber einer Sensitivität von $82 \%$ und einer Spezifität von $57 \%$ für das konventionelle Röntgen [14]. Des Weiteren erleichtert die Kernspintomographie das Auffinden von Abszessformationen und die Abgrenzung gegenüber neoplastischen sowie degenerativen Erkrankungen und ermöglicht eine exakte Beurteilung der Infektionsausdehnung [23]. Insofern wird die MRT als Goldstandard der Bild gebenden Diagnostik der Spondylodiszitis angesehen [19]. Dennoch erlaubt sie keine Differenzierung zwischen spezifischer und unspezifischer Entzündung des Wirbelkörpers und bietet keinen Ersatz für die perkutane diagnostische Biopsie [24]. Mit Ausnahme der Notfallsituationen wie neu aufgetretenen neurologischen Ausfällen und septischen Verläufen stellt somit die CT-gesteuerte Biopsie ein gängiges Verfahren zur Erregerbestimmung dar [17]. Die berichteten Erfolgsraten der Nadelbiopsie werden in der Literatur mit zwischen $50 \%$ und $96 \%$ angegeben [24].

\section{Therapie}

Staphyloccocus aureus ist der häufigste Erreger der unspezifischen und $\mathrm{Myco}$ bacterium tuberculosis der häufigste Erreger der spezifischen Spondylitis/Spondylodiszitis [21]. Die therapeutischen Optionen in der Behandlung der Spondylitis/Spondylodiszitis umfassen konservative sowie operative Maßnahmen. Sie verhalten sich weniger rivalisierend als ergänzend zueinander [23]. Die Ziele der konservativen sowie operativen Therapie sind

- die Schmerzreduktion,

- die Kontrolle der Entzündungsreaktion,

- die schnelle Mobilisation und

- die Vermeidung einer späteren Wirbelsäulendeformität.

\section{Konservative Therapie}

Frühe Stadien ohne wesentliche Destruktion des Wirbelkörpers, ohne Abszessformation, ohne Fehlstellung und ohne neurologische Defizite können konservativ mittels Immobilisation und Gabe von testgerechten Antibiotika therapiert werden. Die Nachteile der konservativen Therapie umfassen eine Immobilisationsphase mit Bettruhe für einen Zeitraum von 7-12 Wochen gefolgt von einer Mobilisation mit Stützkorsett oder Gipsmieder sowie eine unvollständige knöcherne Fusion und kyphotische Fehlstellungen der betroffenen Wirbelsäulensegmente. Hierbei wird über Pseudarthrosen- und Instabilitätsraten von bis zu $50 \%$ berichtet $[9,13]$. Weitere mögliche Nachteile der konservativen Therapie sind die abdominelle Obstruktion durch angelegte Gipsschalen und die ökonomischen und psychosozialen Probleme einer längeren stationären $\mathrm{Be}$ handlung.

\section{Operative Therapie}

Unter Berücksichtigung dieser Gesichtspunkte hat die operative Therapie der Spondylitis/Spondylodiszitis in den letzten Jahren zugenommen [8]. Septische Verläufe sowie neu aufgetretene neurologische Ausfälle stellen Notfälle dar, die einer sofortigen operativen Intervention mit Débridement des Infektherds, Dränage von Abszessen, Dekompressionen und ggf. Stabilisierungen der Wirbel- säule bedürfen. Neben dem septischen Verlauf und neurologischen Ausfällen stellen Abszessformationen, ausgeprägte Wirbelkörperdestruktionen, Fehlstellungen und ein Versagen der konservativen Therapie Operationsindikationen dar.

Die Grundprinzipien der operativen Behandlung sind das Débridement des Infektionsherds kombiniert mit einer Stabilisierung, wodurch eine Korrektur bestehender Deformitäten und eine frühzeitige Mobilisation ohne externe Stabilisationshilfen möglich sind. Ältere Operationstechniken mit Resektion des Infektionsherds und autologer Knochentransplantation benötigten meist postoperative Immobilisationsphasen von mehreren Monaten und externe Stabilisationshilfen in der Nachbehandlung. Neuere operative Techniken mit alleiniger ventraler oder dorso-ventraler Instrumentation mit winkelstabilen Implantaten ermöglichen die frühe postoperative Mobilisation ohne zusätzliches Stützkorsett [8]. Wie in den Fallberichten aufgezeigt, können hiermit ausgeprägte Wirbelkörperdefekte unter Verwendung von Titankörben überbrückt und mit zusätzlichen ventralen Implantaten stabilisiert werden. Hierbei kann in Abhängigkeit vom Grad der Instabilität und der bestehenden Fehlstellung auf eine dorsale Instrumentation verzichtet werden. Ob der Einsatz von Titankörben als Wirbelkörperersatz langfristig zu besseren oder vergleichbaren Ergebnissen wie die Verwendung von autologen kortikospongiösen Spänen führt, ist noch nicht ausreichend geklärt. Vorteile der Titankörbe sind u. a. der Wegfall einer zusätzlichen Morbidität durch die Knochenspanentnahme sowie einer möglichen Spannekrose. Lange Zeit galt der Einsatz von Implantaten in unmittelbarer Nähe des Infektionsherds als kontraindiziert. Ihm wurde eine um 2-9\% erhöhte postoperative Wundinfektionsrate angelastet [15]. In verschiedenen neueren Arbeiten konnten jedoch erhöhte Komplikationsraten bei einzeitigem operativem Vorgehen mit zusätzlicher Instrumentation unter Antibiotikaschutz widerlegt werden [3, 12]. Wir vermeiden es dennoch, bei akuten putriden Verhältnissen ein Implantat einzubringen, und bevorzugen in diesen Fällen ein zweizeitiges Vorgehen mit primärer dorsaler Stabilisierung mit Fixateur interne, Débridement, ggf. Einlage 


\section{Septische Chirurgie}

eines antibiotikahaltigen Platzhalters und sekundärer definitiver ventraler Rekonstruktion.

Da die Spondylitis/Spondylodiszitis den Wirbelkörper und die Bandscheibe betrifft, sollte der operative Zugang gute Übersicht für das Débridement der vorderen Säule schaffen und die stabilisierenden dorsalen Strukturen schonen. Vereinzelt können auch endoskopische Operationstechniken, wie im Fall 5 die thorakoskopische Teilkorporektomie und ventrale Fusion, mit minimierten Zugängen eingesetzt werden.

Eine dekomprimierende Laminektomie bei der Spondylitis/Spondylodiszitis kann zur Instabilität der betroffenen Wirbelsäulensegmente beitragen und $\mathrm{zu}$ Verschlechterungen des neurologischen Status führen $[1,15]$. Kommt es im Verlauf einer Spondylitis/Spondylodiszitis zur epiduralen Abszessbildung, die in etwa 5-18\% der Fälle auftritt, kann die dekomprimierende Laminektomie bei der Entlastung der typischerweise dorsal lokalisierten Abszesse hilfreich sein [1].

\section{Spezielle therapeutische Probleme bei älteren Patienten}

Bei älteren Patienten mit erheblichen Vorerkrankungen müssen bei der Abwägung einer operativen Maßnahme die Nachteile einer verlängerten Bettruhe mit respiratorischen und thrombembolischen Komplikationen sowie Druckulzera und einer erschwerten späteren Mobilisierung berücksichtigt werden. Wie unsere Fallbeispiele zeigen, bevorzugen wir bei entsprechend multimorbiden Patienten die minimalinvasive CT-gesteuerte Abszessdränage und bei bestehender Instabilität die Stabilisierung der betroffenen Wirbelsäulensegmente mit Fixateur interne. Auf größere operative Maßnahmen wie die Herdsanierung und ventrale Fusion wird bei diesen Patienten verzichtet. Speziell bei älteren Patienten mit maskierenden Grunderkrankungen sollte bei unklaren Rückenschmerzen differenzialdiagnostisch früh an eine Spondylitis/Spondylodiszitis gedacht werden, da sich in den Frühstadien konservativ noch gute Ergebnisse erzielen lassen [20].

\section{Resümee}

Trotz flächendeckender Verbreitung der Computertomographie bzw. Kernspintomographie hat die Aussage von Paul Ross aus dem Jahr 1976 [18] weiterhin ihre Berechtigung:

\section{"Neither common enough to be readily recognizable, nor rare enough to be a medical curiosity, vertebral body osteomyelitis represents a diagnostic challenge to the physician."}

\section{Literatur}

1. Abramovitz JN, Batson RA, Yablon JS (1986) Vertebral osteomyelitis - the surgical management of neurologic complications. Spine 11: 418-420

2. Currier BL, Eismont FJ (1992) Infections of the spine. In: Rothmann RH, Simeone FA (eds) The spine. Saunders, Philadelphia, pp 1319-1380

3. Dietze DD, Haid RW (1992) Antibiotic-impregnated methylmethacrylate in treatment of infections with spinal instrumentation: case report and technical note. Spine 17: 981-987

4. Digby W, Kersley JG (1979) Pyogenic nontuberculous spinal infection - an analysis of thirty cases.J Bone Joint Surg Br 61: 47-55

5. Dufek P, Salis-Soglio G, Bozdech Z (1987) Die unspezifische bakterielle Spondylitis - eine Analyse von 32 Fällen.Z Orthop Ihre Grenzgeb 125: $255-261$

6. Flamme $\mathrm{CH}$, Frischalowski T, Gossé $\mathrm{F}$ (2000) Möglichkeiten und Grenzen der konservativen Therapie bei Spondylitis und Spondylodiszitis. Z Rheumatol 59: 233-239

7. Frederickson B, Yuan H, Olans R (1978) Management and outcome of pyogenic vertebral osteomyelitis. Clin Orthop 131: 160-167

8. Hopf C, Meurer A, Eysel P, Rompe JD (1998) Operative treatment of spondylodiscitis what is the most effective approach? Neurosurg Rev 21: 217-225

9. Krödel A, Stürz H (1989) Differenzierte operative und konservative Therapie der Spondylitis und Spondylodiscitis.Z Orthop Ihre Grenzgeb 127: $587-596$

10. Kulowski J (1936) Pyogenic osteomyelitis of the spine.J Bone Joint Surg 18: 343-364

11. Lannelongue OM (1879) On acute osteomyelitis. Pathological and Practical Medicine Tracts 976

12. Levi AD, Dickman CA, Sonntag VK (1997) Management of postoperative infections after spinal instrumentation.J Neurosurg 86: 975 980
13. Medical Research Council Working Party on Tuberculosis of the Spine (1985) A 10-year assessment of controlled trials of inpatient and outpatient treatment and of plaster-of-Paris jackets for tuberculosis of the spine in children on standard chemotherapy. Studies in Masan and Pusan, Korea. Ninth report of the Medical Research Council Working Party on Tuberculosis of the Spine. J Bone Joint Surg Br 67: 103110

14. Modic TM, Feiglin HD, Piraino WD (1985) Vertebral osteomyelitis: assessment using MR. Radiology 157: 157-166

15. Przybylski GJ, Sharan AD (2001) Single-stage autogenous bone grafting and internal fixation in the surgical management of pyogenic discitis and vertebral osteomyelitis.J Neurosurg 94: 1-7

16. Ratcliffe JF (1985) Anatomic basis for the pathogenesis and radiological features of vertebral osteomyelitis and its differentiation from childhood discitis. Acta Radiol 26: 137143

17. Rieneck K, Hansen SE, Karle A, Gutschik E (1996) Microbiologically verified diagnosis of infectious spondylitis using CT-guided fine needle biopsy. APMIS 104: 755-762

18. Ross PM, Fleming JL (1976) Vertebral body osteomyelitis - spectrum and natural history a retrospective analysis of 37 cases. Clin Orthop 118: 190-198

19. Schininà V, Rizzi EB, Rovighi L, Carli $G$ de, David V, Bibbolino C (2001) Infectious spondylodiscitis: magnetic resonance imaging in HIV-infected and HIV-uninfected patients.J Clin Imag 25: 362-367

20. Sindern E, Gläser E, Bötel U, Malin JP (1993) Spondylodiszitis mit spinaler und radikulärer Beteiligung. Nervenarzt 64: 801-805

21. Thrush A, Enzmann D (1990) MR imaging of infectious spondylitis. Am J Neuroradiol 11: 1171-1180

22. Weber M, Heller KD, Wirtz D, ZimmermannPicht S, Keulers P, Zilkens KW (1998) Percutane CT-gesteuerte Punktion und Drainage der Spondylodiszitis - ein minimal invasives Verfahren.Z Orthop Ihre Grenzgeb 136: 375-379

23. Wirtz DC, Genius I, Wildberger JE, Adam G, Zilkens KW, Niethard FU (2000) Diagnostic and therapeutic management of lumbar and thoracic spondylodiscitis - an evaluation of 59 cases. Arch Orthop Trauma Surg 120: 245-251

24. Wood GW (1998) Infections of spine. In: Canale ST (ed) Campbell's operative orthopaedics. Mosby, St Louis, pp 3093-3124 